\title{
Estancia hospitalaria prolongada asociada a mortalidad en Hospital Regional Docente de Trujillo, 2014
}

\section{Prolonged hospital stay associated with mortality in Regional Teaching Hospital in Trujillo, 2014}

\author{
Marie Herrera-Viloche, Edward Chávez-Cruzado, Carlos Barba-Chirinos, Germán Fiestas-Pflucker, Miguel \\ Tresierra-Ayala
}

Universidad Privada Antenor Orrego

DOI: https://doi.org/10.33017/RevECIPeru2015.0010/

\section{Resumen}

La estancia hospitalaria prolongada, considerada aquella que supera los 13 días, genera costos adicionales al hospital, deficiente accesibilidad a los servicios de hospitalización para nuevos pacientes y riesgos de adquirir infecciones intrahospitalarias, así como una crisis social y familiar. Este estudio pretende determinar si la estancia hospitalaria prolongada se encuentra asociada a mortalidad en los pacientes atendidos en las diferentes salas de hospitalización del Hospital Regional Docente de Trujillo en el 2014. Se registraron 11866 hospitalizaciones, y de estos 1193 tuvieron estancia hospitalaria prolongada, de los cuales el $56.58 \%$ fueron de sexo masculino y $43.42 \%$ de sexo femenino. Los departamentos de medicina y cirugía con $45.1 \%$ y $31.3 \%$ y los grupo etarios extremos mayores de 61 y menores de 20 con $29.76 \%$ y $27.75 \%$ fueron los que presentaron estancia hospitalaria prolongada. La asociación pacientes con estancia hospitalaria prolongada y mortalidad, nos da un Odds Radio de 3.276, con IC de 2.615-4.104, con una $p=0.0000$.

Descriptores: Estancia hospitalaria prolongada, mortalidad

\section{Abstract}

Prolonged hospital stay, considered one that exceeds 13 days, generates additional costs to the hospital, poor access to inpatient services for new patients and risk of acquiring nosocomial infections, as well as social and family crisis. This study aims to determine the specialties and age groups associated with prolonged stay of Regional Hospital of Trujillo in 2014. 11866 hospitalizations were recorded, and of these 1193 had prolonged hospital stay, of which $56.58 \%$ were male and $43.42 \%$ female. The departments of medicine and surgery with $45.1 \%$ and $31.3 \%$ and the extreme age group over 61 and under 20 with $29.76 \%$ and $27.75 \%$ were those with longer hospital stay. Patients with prolonged hospital stay and mortality, gives an odds ratio of 3.276, with IC 2615-4104, with $p=0.0000$.

Keywords: prolonged hospital stay

\section{Introducción}

El Hospital Regional Docente de Trujillo (HRDT), es un hospital del Ministerio de Salud nivel III-1, ubicado en la Ciudad de Trujillo, Provincia de Trujillo, Departamento de La Libertad, Peru. Tiene una poblacion referencial de 1, 101,505 y cuenta con 280 camas hospitalarias distribuidas; Cirugia 67, medicina 81 , gineco obstetricia 48 , pediatria 33 , neonatologia 25, UCI 6 y emergencia 20 .

El desarrollo de la gestión sanitaria es uno de los retos al que nos enfrentamos para adaptarnos a las exigencias de calidad y eficiencia. Para ello es muy importante tener un sistema de información que permita medir, evaluar y comparar los resultados de 
todos los procesos asistenciales. La gestión clínica es un método de cambio organizativo y cultural determinado por la descentralización de las decisiones hacia los responsables de las unidades de producción del hospital. Para ello, los médicos deben disponer de los sistemas de información que les permitan demostrar la calidad, los resultados y los costes de sus servicios.

Una forma de evidenciar dichos resultados es el seguimiento a través de la monitorización y control de la estancia hospitalaria, uno de los indicadores clásicos para la valoración de la eficiencia en la gestión de los recursos de los centros hospitalarios. Se considera estancia hospitalaria prolongada a aquella que supera los 13 días [1,2,3].

La estancia hospitalaria prolongada constituye una preocupación mundial, ya que genera efectos negativos en el sistema de salud como, por ejemplo: aumento en los costos, deficiente accesibilidad a los servicios de hospitalización, saturación de las urgencias y riesgos de eventos adversos $[2,4,5]$.

La mortalidad hospitalaria ha sido clásicamente uno de los indicadores de calidad asistencial más frecuentemente utilizado $[6,7,8,9]$. Es el indicador sanitario más antiguo que se conoce, y constituye un valioso instrumento para la planificación y gestión hospitalaria. La duración de la estancia cabe esperar que esté aparejada con la mortalidad, dado que los pacientes con una mayor estancia hospitalaria estarán expuestos a una serie de factores, tales como procedimientos y tratamientos necesarios para su enfermedad pero no exentos de riesgos $[10,11]$.

La contención de costos es una preocupación actual y prioritaria en el contexto de la salud en los países desarrollados, sobre todo en áreas de gastos excesivos con resultados aparentemente insatisfactorios, como lo representan las unidades de terapia intensiva (UTI), donde se llegan a emplear abundantes recursos en la atención de enfermos con bajas probabilidades de sobrevida y una dudosa calidad de vida posterior $[12,13]$.

El análisis de los patrones de empleo de los días en hospitalización es, por lo tanto, un importante paso hacia la optimización de recursos en el área de la medicina y la reducción de la estancia en hospitalización se ha considerado un blanco para los esfuerzos futuros de abatimiento de costos en salud [14], por lo que constituye una necesidad estudiar en profundidad la estancia en estas áreas y, por ende, la mortalidad que nos ayudarían a llevar a cabo una GESTIÓN EFICIENTE del uso de camas de hospitalizazión.

El objetivo del presente estudio es evaluar si la mortalidad se encuentra asociada a la estancia hospitalaria prolongada en los pacientes atendidos en las diferentes salas de hospitalización del Hospital Regional Docente de Trujillo.

\section{Material y método}

Es un estudio de cohorte retrospectivo, con asignación observacional y longitudinal. La población de estudio la constituye todos los pacientes que fueron admitidos en las salas de hospitalización del hospital Regional Docente de Trujillo de enero a diciembre del 2 014. Los cuales cumplían los criterios de inclusión: paciente con estancia mayor a 24 horas, de ambos géneros. Los criterios de exclusión fueron: pacientes con múltiples reingresos, con datos incompletos o ilegibles, egreso por alta voluntaria e ingreso por orden superior. La muestra se recolecto de la base de datos que cumplían los criterios de inclusión y exclusión. Se obtuvieron 11866 pacientes hospitalizados en las diferentes áreas, de los cuales 1193 tenían estancia hospitalaria prolongada y $10 \quad 673$ estancia hospitalaria no prolongada. Para poder contrastar la hipótesis de la presente investigación en las que se la existencia de asociación entre dos variables, se utilizó una serie de estadísticos que permitieron determinar dicha asociación. Como Chi cuadrado $\left(\mathrm{X}^{2}\right)$,esta prueba es a dos colas, considerando un valor $\mathrm{p}<0.05$ como significativo. No se considerará necesario el consentimiento informado dada la naturaleza del estudio y los datos de los pacientes son conocidos únicamente por los médicos investigadores.

\section{Resultados}

Tabla 1. Pacientes hospitalizados con estancia hospitalaria prolongada según sexo, en el HRDT, Enero-Diciembre 2014.

\begin{tabular}{lcccc}
\hline \multirow{2}{*}{ Sexo } & \multicolumn{2}{c}{$\begin{array}{c}\text { ESTANCIA HOSPITALARIA } \\
\text { PROLONGADA }\end{array}$} & \multicolumn{2}{c}{$\begin{array}{r}\text { ESTANCIA HOSPITALARIA } \\
\text { NO PROLONGADA }\end{array}$} \\
\cline { 2 - 5 } & $\mathrm{N}$ & $\%$ & $\mathrm{~N}$ & $\%$ \\
\hline Femenino & 518 & 43.42 & 7281 & 68.22 \\
Masculino & 675 & 56.58 & 3392 & 31.78 \\
\hline TOTALES & 1193 & 100.00 & 10673 & 100.00 \\
\hline
\end{tabular}

Fuente: Oficina de Estadística y Epidemiología 


\begin{tabular}{cccc}
\hline \multirow{2}{*}{$\begin{array}{c}\text { ESTANCIA } \\
\text { HOSPITALARIA }\end{array}$} & \multicolumn{2}{c}{$\begin{array}{c}\text { MORTALIDAD } \\
\text { HOSPITALARIA }\end{array}$} & TOTAL \\
\cline { 2 - 3 } & MUERTO & VIVO & \\
\hline ESTANCIA PROLONGADA & $110 \mathrm{a}$ & $1083 \mathrm{~b}$ & 1193 \\
$\begin{array}{c}\text { ESTANCIA NO } \\
\text { PROLONGADA }\end{array}$ & $321 \mathrm{a}$ & $10352 \mathrm{~b}$ & 10673 \\
\hline Total & 431 & 11435 & 11866 \\
\hline
\end{tabular}

Tabla 2. Estancia hospitalaria prolongada según especialidades en HRDT enero-diciembre 2014.

\begin{tabular}{|c|c|c|c|c|}
\hline \multirow[t]{2}{*}{$\begin{array}{c}\text { AREA DE } \\
\text { HOSPITALIZACIÓN }\end{array}$} & \multicolumn{2}{|c|}{$\begin{array}{c}\text { ESTANCIA } \\
\text { HOSPITALARIA } \\
\text { PROLONGADA } \\
\end{array}$} & \multicolumn{2}{|c|}{$\begin{array}{c}\text { ESTANCIA } \\
\text { HOSPITALARIA NO } \\
\text { PROLONGADA } \\
\end{array}$} \\
\hline & $\mathrm{N}$ & $\%$ & $\mathrm{~N}$ & $\%$ \\
\hline CIRUGIA & 373 & 31.3 & 2747 & 25.7 \\
\hline GINECOSBTETRICIA & 35 & 2.9 & 4089 & 38.3 \\
\hline MEDICINA & 538 & 45.1 & 2456 & 23.0 \\
\hline PEDIATRIA & 74 & 6.2 & 614 & 5.8 \\
\hline UCI MEDICINA & 19 & 1.6 & 36 & .3 \\
\hline UCI NEONATAL & 149 & 12.5 & 725 & 6.8 \\
\hline Total & 1193 & 100.0 & 10673 & 100.0 \\
\hline \multirow[t]{2}{*}{ Grupo Etario } & \multicolumn{2}{|c|}{$\begin{array}{c}\text { ESTANCIA } \\
\text { HOSPITALARIA } \\
\text { PROLONGADA } \\
\end{array}$} & \multicolumn{2}{|c|}{$\begin{array}{c}\text { ESTANCIA } \\
\text { HOSPITALARIA NO } \\
\text { PROLONGADA } \\
\end{array}$} \\
\hline & $\mathrm{N}$ & $\%$ & $\mathrm{~N}$ & $\%$ \\
\hline 0-20 años & 331 & 27.75 & 3168 & 29.68 \\
\hline 21-40 años & 280 & 23.47 & 4140 & 38.79 \\
\hline 41-60 años & 227 & 19.03 & 1533 & 14.36 \\
\hline $61-80$ años & 251 & 21.04 & 1350 & 12.65 \\
\hline 81-104 años & 104 & 8.72 & 482 & 4.52 \\
\hline TOTALES & 1193 & 100 & 10673 & 100 \\
\hline
\end{tabular}

Tabla 3. Estancia hospitalaria prolongada según grupos etarios. HRDT, enero-diciembre 2014

Tabla 4. La mortalidad entre pacientes de estancia prolongada versus no prolongada
Tabla 5. Pruebas de chi-cuadrado para coparar variables

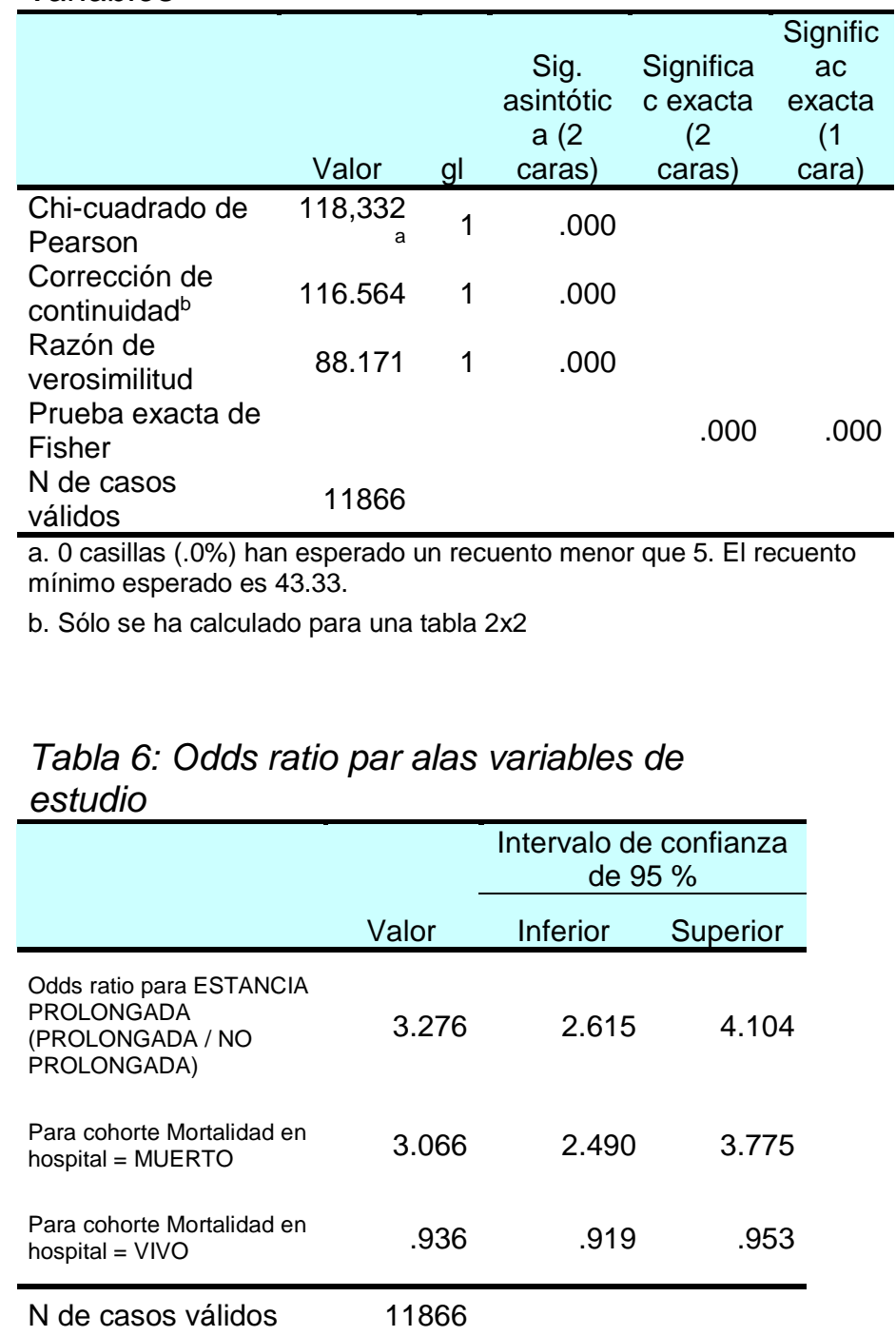

\section{Discusión}

Se registraron 11866 hospitalizaciones, y de estos 1 193 tuvieron estancia hospitalaria prolongada, de los cuales el $56.58 \%$ fueron pacientes de sexo masculino y $43.42 \%$ de sexo femenino, los departamentos de medicina y cirugía con $45.1 \%$ y $31.3 \%$ y los grupo etarios extremos mayores de 61 y 
menores de 20 con $29.76 \%$ y $27.75 \%$ fueron los que presentaron mayor estancia hospitalaria Tabla 3.

Referente a la edad estos resultados son similares al estudio de M. Camacho [4] y C. De Dios et al. [15] en donde encontraron como factor de riesgo de estancia hospitalaria prolongada a la edad avanzada. Con los resultados que se muestran en la Tabla 1, se evidencia que al sexo masculino es el que predomina al igual que los estudios por $\mathrm{C}$. Zamudio et al. [16] y Santana et al. [17]. Otra de las variables estudiadas es el área de hospitalización, en donde se evidencia que los pacientes de medicina y cirugía son lo más frecuentes Tabla 2 , ésto puede deberse a la hospitalización de la parturienta en el área de obstetricia; resultados similares se observan en los estudios de A.G. Stack et al. [18] y E.A. Jackson et al. [19], en donde además los pacientes que tiene estancia prolongada son pacientes con patología crónicas.

La mortalidad encontrada en el presente estudio en pacientes con estancia prolongada fue de $9.2 \%$ como se muestra en la Tabla 4, similares resultados son reportados por R.M. Lorenzo [1] pero difieren de C. Zamudio et al. [16]; que presentan valores menores, esto pueda deberse posiblemente a que en nuestro estudio se ha tomada en cuenta a la mortalidad en general mientras que en él solo considero al departamento de enfermedades infecciones y tropicales, dermatológicas. Así mismo se encontró en nuestro estudio que existe una diferencia significativa entre la mortalidad de paciente con estancia prolongada y estancia no prolongada, como muestra la Tabla 6; que difieren con C.L. Santana et al. [17] y se debería probablemente a que su población de estudio son paciente de $\mathrm{UCl}$ adultos.

Ambas variables están asociadas estadísticamente como vemos en la Tabla 6 , el valor $p$ de la prueba Chi de Pearson es $<0.000001$.

Para determinar si existe relación de riesgo entre la estancia prolongada y la mortalidad en la Tabla 7, se observa que la Odds Radio es de 3.276, con IC de 2.615-4.104, con una $p=0.0000$; lo que nos indica que pacientes con estancia hospitalaria prolongada constituye tienen 3.276 veces mayor riesgo de mortalidad al ser comparados con pacientes con estancia hospitalaria no prolongada; resultados que son similares a los de K.B. Laupland et al. [20]. Con lo que podremos afirmar que existe reación de riesgo entre la estancia hospitalaria prolongada y la mortalidad del paciente en el ambiente hospitalario.

\section{Conclusiones}

En estudio de la estancia hospitalaria en el Hospital Regional Docente de Trujillo, podemos concluir que la mortalidad está relacionada con la estancia hospitalaria prolongada, constituyendo ésta, un factor de riesgo de muerte intrahospitalaria que aumenta en tres veces el riesgo de muerte cuando un paciente excede los 13 días de estancia.

La estancia hospitalaria prolongada está relacionada con el sexo masculino y con las hospitalizaciones en las áreas de Medicina y Cirugía.

\section{Referencias}

[1] R.M. Lorenzo, Análisis comparativo del perfil y la mortalidad de los pacientes con estancia intermedia versus prolongada en cuidados intensivos en función del tipo de paciente. \{tesis doctoral\}. Facultad de ciencias de la salud departamento de ciencias médicas y quirúrgicas: Universidad de las Palmas de Gran Canaria;2014.

[2] I. González, Relación entre el prestador de servicio de salud y la estancia prolongada en el hospital. Revista Conamed. 14 (2009) 4.

[3] T. Ceballos-Acevedo, P.A. VelásquezRestrepo, J. Jaén-Posada, Duración de la estancia hospitalaria. Metodologías para su intervención. Rev. Gerenc. Polít. Salud. 13 (2014) 274-295. http://dx.doi. org/10.11144/Javeriana.rgyps13-27.dehm

[4] M.E. Camacho, Estancia prolongada de pacientes en paciente post operados. Revista salud, sexualidad y sociedad. 2 (2009) 2-9.

[5] M.S. Peiró, E. Portella, Identificación del uso inapropiado de la hospitalización: la búsqueda de la eficiencia. Med Clin (Barc). 103(1994) 65-71.

[6] A. Pérez-Rubio, S. Santos, F.J. Luquero, S. Tamames, B. Cantón, J.J. Castrodeza, Evaluación de la adecuación de las estancias en un hospital de tercer nivel. An Sist Sanit Navar. 30(2007) 29-36.

[7] M.S. McDonagh, D. Smith, M. Goddard, Measuring appropriate use of acute beds. A systematic review of methods and results. Health Policy. 53(2000) 157-84.

[8] S. Peiró, R. Meneu, M.L. Roselló, E. Portella, R. Carbonell-Sanchís, C. Fernández, et al. Validez del protocolo de evaluación del uso inapropiado de la hospitalización. Med Clin (Barc). 107(1996) 124-9.

[9] D. Fuente, S. Peiró, E. Portella, C. Marchan, S. Aymerich, Utilización innecesaria de la 
hospitalización: Importancia de la gestión a nivel de Servicio. Rev Calidad Asistencial. 1(1994) 8-16.

[10] I. Strumwasser, N.V. Paranjpe, D.L. Ronis, D. Share, L.J. Sell, Reliability and validity of utilization review criteria. Appropriateness Evaluation Protocol, standardized medreview instrument, and intensity-severity-discharge criteria. Med Care. 28(1990) 95-111.

[11] P.A. Lipsett, S.M. Swoboda, J. Dickerson, M. Ylitalo, M. Gordon, M. Breslow, K. Campbell, T. Dorman, P. Pronovost, B. Rosenfeld, Survival and functional outcome after prolonged intensive care unit stay. Ann Surg. 231(200) 262-8

[12] A.L. Rosenberg, J.E. Zimmerman, C. Alzola, E. Draper, W. Knaus, Intensive care unit length of stay: recent changes and future challenges. Crit Care Med. 28(2000) 3465-73

[13] N.A. Halpern, L. Bettes, R. Greenstein, Federal and nationawide intensive care unit and healthcare costs: 1986-1992. Crit Care Med 22(1994) 2001-7.

[14] D.B. Chalfin, I.L. Cohen, J. Lambrinos, The economics and cost effectiveness of critical care medicine. Intensive Care Med 21(1995) 52-61.

[15] C. De Dios, C. López, D. Damnie, V. Rodríguez, Morbilidad y mortalidad en pacientes egresados de la unidad de cuidados intensivos de Contramaestre durante un bienio. Rev Calidad Asistencial. 5(1999) 1823.

[16] C. Zamudio, R.C. Seas, M.K. Hernandez, S.E. Ramos, B.K. Verdonck, H.R. Chuquiyauri, Morbilidad y mortalidad en el servicio de hospitalización del Departamento de enfermedades infecciosas, tropicales y dermatológicas del Hospital Nacional Cayetano Heredia entre 1990 - 2000. Informe del Servicio de Enfermedades Infecciosas y Tropicales. Hospital Nacional Cayetano Heredia. 2002.
[17] C.L. Santana, T.M. Lorenzo, P. Sanchez, J. Santana, R. Hernandez, Pronóstico de los pacientes médicos según la duración de su estancia en la unidad de cuidados intensivos. Med Intensiva.

2013. http://dx.doi.org/10.1016/j.medin.2013.06.004

[18] A.G. Stack, W.E. Bloembergen, Prevalence and clinical correlates of coronary artery disease among new dialysis patients in the United States: a cross sectional study. Journal of the American Society of Nephrology. 12(2001) 1516-23.

[19] E.A. Jackson, M. Moscucci, D.E Smith, D. Share, S. Dixon, A. Greenbaum, E.A. Grockson, M. Moscucci, P.M. Grossman, H.S. Gurm. The association of sex with outcomes among patients undergoing primary percutaneous coronary intervention for ST elevation myocardial infarction in the contemporary era: Insights from the BlueCross Blue Shield of Michigan Cardiovascular Consortium (BMC2). Am Heart J. 161(2011) 106-12.

[20] K.B. Laupland, A.W. Kirkpatrick, J.B. Kortbeek, D.J. Zuege, Long-term mortality outcome associated with prolonged admission to the ICU. Chest. 129(2006) 954-9.

E-mail:mtresierra@medicos.com 